\title{
Gestión organizacional y productividad laboral en las unidades educativas en el Sur De Manabí De Ecuador
}

\section{Organizational management and labor productivity in educational units in southern Manabí, Ecuador}

\author{
DOI: $10.46932 / \mathrm{sjjdv2n4-003}$
}

Received in: March 1st, 2021

Accepted in: May 30th, 2021

\section{Ing. Maribel Celi Vásquez Paucar}

PhD. Docente de la Universidad Estatal del Sur de Manabí; Jipijapa - Manabí - Ecuador, Km 1 1² vía a Noboa; Estudiante de Doctorado en Gestión Empresarial de la Universidad La Habana - Cuba correo electrónico: maribelceli@yahoo.com; maribel.vasquez@unesum.edu.ec

\section{Ing. Zoe Rodríguez Cotilla}

$\mathrm{PhD}$. Docente de la universidad de La Habana - Cuba y Coordinadora del Doctorado en Administración y Gestión Empresarial de la Universidad de la Habana - Cuba - El Vedado - Calle L No. 353 entre 21 y 23

correo electrónico zoe@ fec.uh.cu

\section{Ing. Oreste La O León}

$\mathrm{PhD}$. Docente y Coordinador de la oficina de Evaluación y Aseguramiento de la Calidad de la Universidad Nacional de Loja - Ecuador - Av. Pio Jaramillo Alvarado, Loja 110103 correo electrónico: orestes.lao@gmail.com; oreste.lao@unl.edu.ec

\section{Eco. Miguel Ángel Padilla Orlando}

Magister en Contabilidad y Auditoría, Docente de la Universidad Estatal del Sur de Manabí - Jipijapa Manabí - Ecuador, Km 1 1⁄2 vía a Noboa; correo electrónico: miguel08012011@ @otmail.com; miguel.padilla@unesum.edu.ec

\section{RESUMEN}

Con el objetivo de conocer la gestión organizacional y la dinámica de productividad laboral del talento humano para el funcionamiento y evolución de las instituciones educativas del Distrito 13D03 de la zona 4 en Jipijapa - Manabí, se realizó un estudio para identificar si se aplican o no los procesos administrativos, si la gestión realizada en la organización es correcta, y con ello se logra la productividad laboral del talento humano. La metodología utilizada en la investigación fue cualitativa - cuantitativa, las técnicas aplicadas fueron: encuestas al personal docente, administrativo y de servicio, y la entrevista, a los directivos de los planteles educativos. El número de la muestra correspondió a 536 personas. En los resultados obtenidos, se determinó que no se está llevando de forma eficiente la gestión organizacional, y con ello el talento humano se siente insatisfecho por la no aplicación de los reglamentos en la aplicación de planes de incentivos económicos o no económicos, controles inadecuados de horarios de trabajo, sueldos y salarios indignos, carencia de capacitaciones en áreas administrativas, así como en desarrollo personal, incorrecta comunicación, deficiente colaboración de equipos de trabajo, y demás deberes y derechos del trabajador. Por lo que se concluye que la inexistencia de un modelo de gestión organizacional ajustado a las necesidades del entorno, influye de manera directa en la productividad de quienes laboran en las unidades educativas.

Palabras claves: organización, gestión, rendimiento profesional, educación media, docentes. 


\begin{abstract}
With the objective of knowing the organizational management and the dynamics of labor productivity of human talent for the operation and evolution of the educational institutions of District 13D03 of zone 4 in Jipijapa - Manabí, a study was carried out to identify whether or not the administrative processes, if the management carried out in the organization is correct, and with this the labor productivity of human talent is achieved. The methodology used in the research was qualitative - quantitative, the techniques applied were: surveys of the teaching, administrative and service personnel, and the interview with the directors of the educational establishments. The number of the sample corresponded to 536 people. In the results obtained, it was determined that the organizational management is not being carried out efficiently, and with this human talent feels dissatisfied by the non-application of the regulations in the application of economic or non-economic incentive plans, inadequate controls of unworthy working hours, wages and salaries, lack of training in administrative areas, as well as in personal development, incorrect communication, poor collaboration of work teams, and other duties and rights of the worker. Therefore, it is concluded that the lack of an organizational management model adjusted to the needs of the environment directly influences the productivity of those who work in educational units.
\end{abstract}

Keywords: organization, management, professional performance, secondary education, teachers.

\title{
1 INTRODUCCIÓN
}

A nivel mundial la educación es considerada el motor del progreso; y al ser administrada correctamente con sentido de pertinencia y trabajo social, se logra la correcta administración y gestión de los procesos, que permiten cumplimiento de la misión y objetivos de la institución. En este sentido los gobiernos de turno asignan presupuestos acordes a las exigencias y avances de las tecnologías, para hacer frente a la nueva normalidad que son los entornos virtuales, aplicados en las IES de manera emergente por la presencia de la pandemia del COVID 19 y con miras a fortalecer la impartición de clases, se cuenta con personal científico especializado para alcanzar y crear los más grandes avances de la historia, que fortalecen las funciones sustantivas de la educación: docencia, investigación, vinculación y gestión administrativa.

Dentro de este orden de ideas, organizaciones como la UNESCO reconocen, que la lucha por una educación inclusiva y de calidad se basa en el derecho de todos y todas a recibir una educación que promueva el aprendizaje durante toda la vida. Un sistema educativo es de calidad cuando presta atención a los grupos marginados y vulnerables y procura desarrollar su potencial. (UNESCO, 2017, p. 1).

Concerniente a ello, el Banco Mundial (2017), exterioriza que "La educación conlleva beneficios considerables y sistemáticos en materia de ingresos, y contrarresta el aumento de la desigualdad” (p. 1).

Desde la antigüedad, la administración en las empresas ha sido fundamental para que estas tengan el éxito requerido, la gestión organizacional es un factor importante que se debe de considerar para el desarrollo de las entidades públicas o privadas, más aún cuando está encaminado a determinar la potencialidad que posee cada uno de sus colaboradores. Con la correcta aplicación del sistema de gestión 
organizacional se logrará medir la productiva laboral, determinando así la eficiencia, eficacia y efectividad que cada persona entrega en la entidad, con el afán de demostrar un trabajo de calidad que se convierta en su carta de presentación.

En la provincia de Manabí, las entidades educativas de nivel medio, son organismos que se encargan de la educación de los jóvenes y adolescentes, quienes representan el futuro de las naciones. En ellos se encuentran laborando un gran número de profesionales, mismos que demuestran su capacidad intelectual, y entregan toda su sapiencia con el ánimo de lograr mejores días para la humanidad, los directivos de las unidades educativas tienen la misión de implantar un clima laboral y una cultura organizacional adecuada para lograr en mayor porcentaje la productividad profesional de sus colaboradores.

La gestión organizacional encaminada a medir la productividad laboral en las unidades educativas del sur de Manabí, permite detectar anomalías o buenos resultados en las diversas áreas que conforman la organización.

El trabajar con talento humano es una tarea muy ardua, ya que cada ser humano es un mundo que tiene diferentes actitudes y aptitudes y estos hacen de ellos seres especiales y extraordinarios, así como de incrementar sus ganas de superación personal o familiar.

En los últimos treinta años, el recurso humano, ha ido ocupando un lugar preponderante en las organizaciones, por su decisivo rol, tanto en los resultados productivos o de servicios, como en sus aportes en las innovaciones y en el desarrollo global de la gestión, cada una de ellos con el firme propósito de contribuir al desarrollo educativo, económico, social y cultural del país.

En base a lo descrito es fundamental el amplio estudio de la gestión organizacional y la productividad laboral, variables del presente estudio, el que tiene su basamento en diversas teorías, tales como:

Frederick Taylor (1969) sostenía que el éxito de los principios requería una "revolución total de la mentalidad" de los obreros y patrones. En lugar de pelearse por las utilidades, las dos partes deberían poner su empeño en elevar la producción, y en su opinión, al hacerlo, las utilidades aumentarían a tal grado que los obreros y los patrones ya no tendrían que pelearse por ellas. En pocas palabras, Taylor pensaba que tanto obreros como patrones tenían el mismo interés en elevar la productividad.

En referencia a lo descrito, en el año 2008 en Ecuador, el Ministerio de Educación, aplicó pruebas diagnósticas a nivel nacional a docentes y estudiantes de la educación fiscal, en donde se evidenció un exiguo nivel de rendimiento, también que las políticas educativas del Ministerio de Educación estaban descontextualizadas de la realidad educativa del país y que modelos pedagógicos impuestos no habían respondido a la necesidad local y nacional, "Ya que cada vez se acercaron más a una perspectiva 
empresarial y se alejaron de una comprensión pedagógica del proceso de enseñanza aprendizaje”

Referirse a cerca de gestión organizacional y productividad laboral es describir el trabajo desplegado en el ambiente interno y externo de la organización, porque la eficiente labor permite alcanzar los estanderes de calidad que necesita cada producto o servicio ofertado y por ende la institución en su totalidad. Por lo descrito, y por la importancia del tema, se acentua lo significativo del estudio y, se propone el siguiente objetivo: Conocer la gestión organizacional y la dinámica de productividad laboral del talento humano para el funcionamiento y evolución de las instituciones educativas de la zona 4 en el sur de Manabí

\section{MARCO TEÓRICO}

En la actualidad las concepciones administrativas son la resultante de un proceso iniciado en los mismos albores de la humanidad y que ha venido evolucionando y adquiriendo sus propios perfiles a través de diferentes épocas y etapas.

En el siglo XX, se consolida y desarrolla la administración como ciencia indispensable para el desarrollo industrial y económico, además es necesario destacar que surgen nuevos enfoques y escuelas para el estudio de esta materia.

Autores como Blanco, Samaco y Berrelleza (2018) sustentan "La administración es una actividad que llevamos a cabo los seres humanos en diferentes ámbitos de nuestra vida diaria, se considera como un área de conocimientos necesarias para el hombre" (P.9).

En el marco de este concepto, administrar es aplicar un sistema de trabajo que permite lograr el desempeño de objetivos y metas planteados.

Con relación a la temática, Munch (2014) precisa. "El proceso administrativo es una metodología que permite al administrador, gerente, ejecutivo, empresario o cualquier otra persona, manejar eficazmente una organización” (P.15).

El proceso administrativo está conformado por 4 etapas, como son: planeación, organización, dirección y control.

López (2019) resalta que "La planeación, trata de programar las tareas a realizar y resolver las cuestiones pertinentes que más tarde se ejecutarán (P.1).

En la dirección, se encuentra la ejecución de los planes, la motivación, la comunicación y la supervisión para alcanzar las metas de la organización, siendo el control la función que se encarga de evaluar el desarrollo de lo planeado y lo ejecutado (Carrasco y Viñán, 2017 p.15)

Siendo la organización la segunda etapa del proceso administrativo y uno de los elementos esencial de la fase estática, es el punto central e importante del presente estudio, por ello se enfatiza en su 
conceptualización. El investigador Ramírez (2015) acentúa "La organización consiste en el diseño y determinación de las estructuras, procesos, funciones y responsabilidades, así como el establecimiento de métodos, y la aplicación de técnicas tendientes a la simplificación del trabajo" (P.25).

Por otra parte, las ciencias administrativas o gestión tienen diversos aspectos u obstáculos epistemológicos en la construcción de un nuevo conocimiento como lo enuncia a continuación Soto:

a. La ausencia y carencia de reflexión teórica en el campo.

b. La aplicación de técnicas de investigación bajo criterios de usos y costumbres de la "ciencia", sin cuestionarse sobre la pertinencia en los objetos de estudios. (Contreras Soto, 2011)

En la época primitiva, al trabajar el hombre en grupo surgió de manera incipiente la administración. Con la aparición del Estado, que señala el inicio de la civilización, surgieron la ciencia, la literatura, la religión, la organización política, la escritura y el urbanismo. En la antigua Grecolatina, apareció el esclavismo, durante el cual la administración se orientaba hacia una estricta supervisión del trabajo. En el transcurso del feudalismo, las relaciones sociales se caracterizaron por un régimen de servidumbre, en el siglo XX se distingue un gran desarrollo tecnológico e industrial y en consecuencia por la consolidación de la administración, surge la administración científica cuyo iniciador fue Taylor, (Munch, 2014).

Antes de centrar la investigación en la productividad laboral y sus factores, es lógico e imprescindible realizar un recordatorio a cerca de las teorías que fundamentan el tema, para ello se inicia con la conceptualización de Gestión del talento humano, ya que es la matriz desde donde nace la labor con las personas. El término Recursos Humanos fue acentuado por Rucker, 1954, como una de las funciones clave de la Administración de Empresas, asi Chiavenato, demuestra que la gestión del talento humano es un área muy sensible a la mentalidad que predomina en las organizaciones. (Chiavenato, 2008).

\section{PRODUCTIVIDAD LABORAL}

En la actualidad, para obtener una posición competitiva, las empresas necesitan orientarse hacia todos los niveles de la estructura organizativa, requiriéndose entonces una nueva visión de gerente, el cual debe buscar integrar a las personas, los procesos y las tecnologías administrativa, en las instituciones del sector público, comienza a tomar gran importancia a partir de la crisis económica de la década de los 80 , también llamada "crisis de la deuda latinoamericana de los 80". La crisis de la deuda. (FrenchDavisy1993).

Descritos los conceptos generales, y centrando el estudio de temas específicos, hablar de productividad laboral es describir el trabajo que realiza el ser humano en una organización. Para (ACEDO, 2011). El término productividad, es un concepto que se utiliza en las grandes empresas y organizaciones para contribuir a la mejora de la productividad, por ello muchos autores han estudiado sobre la temática 
y es así que para Garza, 2006, la productividad laboral se puede definir como la relación existente entre la producción de bienes y servicios con las cantidades de insumos, trabajo y capital utilizado (p.13).

Mientras que Koopmans (citada en Gabini y Salessi, 2018), afirma que "El rendimiento laboral (RL) es uno de los constructos que mayor atención ha recibido por parte de los estudiosos del comportamiento organizacional. Posiblemente, su popularidad se deba a que la competitividad y la productividad de las organizaciones se encuentran íntimamente ligadas al desempeño individual de sus miembros" (p. 32).

Mientras que para el Sistema de Producción de Productividad Laboral (2012), en términos económicos, la productividad reduce los costos de producción y eleva la rentabilidad de las inversiones. En términos generales, el incremento de la productividad "Puede contribuir a aumentar los ingresos y reducir la pobreza, generando así un círculo virtuoso. En efecto, el aumento de la productividad reduce los costos de producción y aumenta rentabilidad de las inversiones; una parte se convierte en ingresos para los propietarios de las empresas y los inversionistas, y otra parte se convierte en aumentos salariales." (p.8)

Según Velázquez (2016), la productividad se ha convertido en el principal problema de la mayoría de las empresas, puesto que existen muchas situaciones internas que afectan el rendimiento de los empleados y que muy pocos se han detenido a solucionarlas, y refiere algunos factores que considera afectan la productividad laboral:

1. Jornadas laborales largas.

2. Falta de capacidad laboral.

3. Actividades multitareas.

4. Períodos de descanso cortos.

5. Malas condiciones laborales (p. 1)

Atendiendo a los criterios de Word Meter en su libro, Ladrones del tiempo, (2012), describe detalladamente elementos del por qué no se logra la productividad laboral, y considera, existen dos tipos de factores, internos y externos.

Para él, los factores externos, son un conjunto de circunstancias que exceden la capacidad de control del individuo, entre ellos se encuentran: el e-mail o correo electrónico, el teléfono, servicios de mensajería instantánea, reuniones de trabajo, e interrupciones.

Mientras que considera, que dentro de los factores internos, los más significativos son: los hábitos multitareas, la precaria planificación, carencia de objetivos, falta de motivación, la dificultad para dar una réplica negativa (p.4). 
La productividad laboral, es la medida en la que un trabajo se convierte en una cantidad de bienes o servicios; es la relación entre la producción alcanzada y el uso eficiente de todos los recursos, tiene estrecha relación con la eficiencia y el beneficio; si se relaciona con valor de uso y período de tiempo; lo que está relaciona a la capacidad, competencias y conocimientos que posee y emplea el ser humano, para desarrollar de forma idónea las tareas u objetivos encargados.

Desde esta investigación se reconoce que la productividad es la relación-resultado e insumo dentro de un período con la debida consideración de la calidad, se puede expresar de la forma siguiente:

1. Al acrecentar la producción (resultados) con los mismos insumos.

2. Al disminuir los insumos manteniendo la misma producción.

3. Al aumentar la producción y disminuir los insumos para cambiar la razón de un modo favorable.

\section{TEORÍA DE MASLOW SOBRE LA JERARQUÍA DE LAS NECESIDADES HUMANAS.}

Según Maslow, las necesidades humanas están organizadas en una pirámide de acuerdo con su importancia respecto a la conducta humana.

1. Necesidades fisiológicas: constituyen el nivel más bajo de necesidades humanas. Son necesidades innatas, como la necesidad de alimentación (hambre o sed), sueño y reposo (cansancio), abrigo (contra frío o calor) o deseo sexual (reproducción de la especie).

2. Necesidades de seguridad: constituyen el segundo nivel en las necesidades humanas. Llevan a las personas a protegerse de cualquier peligro real o imaginario, físico o abstracto.

3. Necesidades sociales: son las necesidades que surgen de la vida social del individuo con otras personas. Son las necesidades de asociación, participación, aceptación por parte de sus compañeros, intercambio de amistad, afecto y amor.

Además, las otras necesidades, una vez satisfechas, ya no motivan la conducta, en cambio, la necesidad de autorrealización puede ser insaciable, en el sentido de que entre más satisfacción obtiene (Maslow, 1957 - 2007).

\section{TEORÍA DE LOS DOS FACTORES DE HERZBERG}

La teoría de los dos factores de Herzberg Mientras Maslow apoya su teoría de la motivación en las diferentes necesidades humanas (perspectiva introvertida), Herzberg basa su teoría en el ambiente externo (perspectiva extravertida). Para Herzberg, la motivación para trabajar depende de dos factores: 
FACTORES HigiÉNICOS O FACTORESEXTRÍNSECOS, están relacionados con la insatisfacción, pues se localizan en el ambiente que rodean a las personas y abarcan las condiciones en que desempeñan su trabajo. Como esas condiciones son administradas y decididas por la empresa, los factores higiénicos están fuera del control de las personas. Los principales factores higiénicos son:

Sueldos y salarios

Beneficios sociales

Dirección o supervisión

Condiciones físicas y ambientales

Políticas y directrices

Clima de relaciones

FACTORES MOTIVACIONALES O FACTORESINTRÍNSECOS, están relacionados con la satisfacción en el cargo y con la naturaleza de las tareas que el individuo ejecuta, son controlados por el individuo, se relacionan con aquello que él hace y desempeña, involucran los sentimientos relacionados con el crecimiento y desarrollo personal, el reconocimiento profesional, las necesidades de autorrealización, la mayor responsabilidad y dependen de las tareas que el individuo realiza en su trabajo. (Chiavenato 1996 - 2007)

En contexto, con las conceptualizaciones descritas, se presentan por parte del Ministerio de Educación (2017), dimenciones que los directivos deben cumplir para dirigir de forma armoniosa una organización:

Dimensión A: Planificación Estratégica

Dimensión B: Gestión Pedagógica

Dimención C: Gestión del Talento Humano y Recursos

Dimensión D: Clima Organizacional y convicencia escolar

De la misma forma el Ministerio de educación, presenta el manual para la implementación de estandares de calidad, el que tiene como objetivo, orientar, apoyar y monitorear la acción de los grupos de actores que conforman el Sistema Nacional de Educación para su mejora continua. (Manual, 2017. p.14).

Con este radio de acción los directivos nacionales, apuntan a mejorar la gestión en las instituciones educativas y con ello mejorar la productividad laboral, para entregar a la sociedad profesionales con calidad y calidez. 
Estudios realizados a nivel mundial, comprueban que la relación entre gestión y las tres dimensiones de la productividad (Eficiencia, Eficacia y Competitividad), son indispensables e imprescindibles, por que demuestran que las empresas se encuentran en procesos cambiantes, propios del mundo globalizado, es por ello que se requiere la obtención de nuevos conocimientos y habilidades que permitan innovar para lograr posiciones competitivas con el apoyo de los sistemas de información( (Avalos, 2021)

Bajo el contexto de busqueda de mejoras para la educación media, y aplicando la innovación técnologica (Castro, 2021), menciona que la necesidad del siglo XXI, obliga a que la educación se brinde en nuevas modalidades, rompiendo las barreras del tiempo y el espacio, conllevando a la creación de programas virtuales que cumplan los estándares de calidad requeridos y con ello dinamizar la gestión de los procesos en las unidades educativas.

\section{METODOLOGÍA}

En el desarrollo de la investigación se aplicaron métodos y técnicas que contribuyeron al avance y recogida de información.

Dentro de los métodos utilizados se encuentra el cualitativo - cuantitativo, los que se cumplieron con las bases teóricas de las variables, y resultados obtenidos de las técnicas aplicadas como son: encuestas (personal docente, administrativo y de servicio), y la entrevista (directivos de los planteles educativos). Determinando para ello una muestra de 536 personas.

\section{RESULTADOS.}

Los resultados son el proceso obtenido del dialogo, la observación directa y las encuestas que se aplicaron a los directivos, docentes y administrativos de las entidades educativas, puntualizando que las variables objeto de estudio fueron la Gestión organizacional y la productividad laboral. Siendo la gestión organizacional el proceso de diseñar y mantener un ambiente en el cual los individuos, trabajan en grupo de forma eficiente para alcanzar los objetivos planteados (Koonts, 2012)

\section{Encuestas personal administrativo:}

Para conocer si se aplican o no las etapas del procesos administrativos, se pretende abordar dos variables de interés como son: Productividad laboral y gestión organizacional, el estudio de ellas permitirá conocer el accionar del elemento humano y con ello la dinámica de la gestión para el correcto funcionamiento y evolución de las instituciones. Al hacer las preguntas: 
¿Conoce usted las etapas del proceso administrativo? y ¿Mencione cuál de ellas aplica? (gráfico 1 y 2)
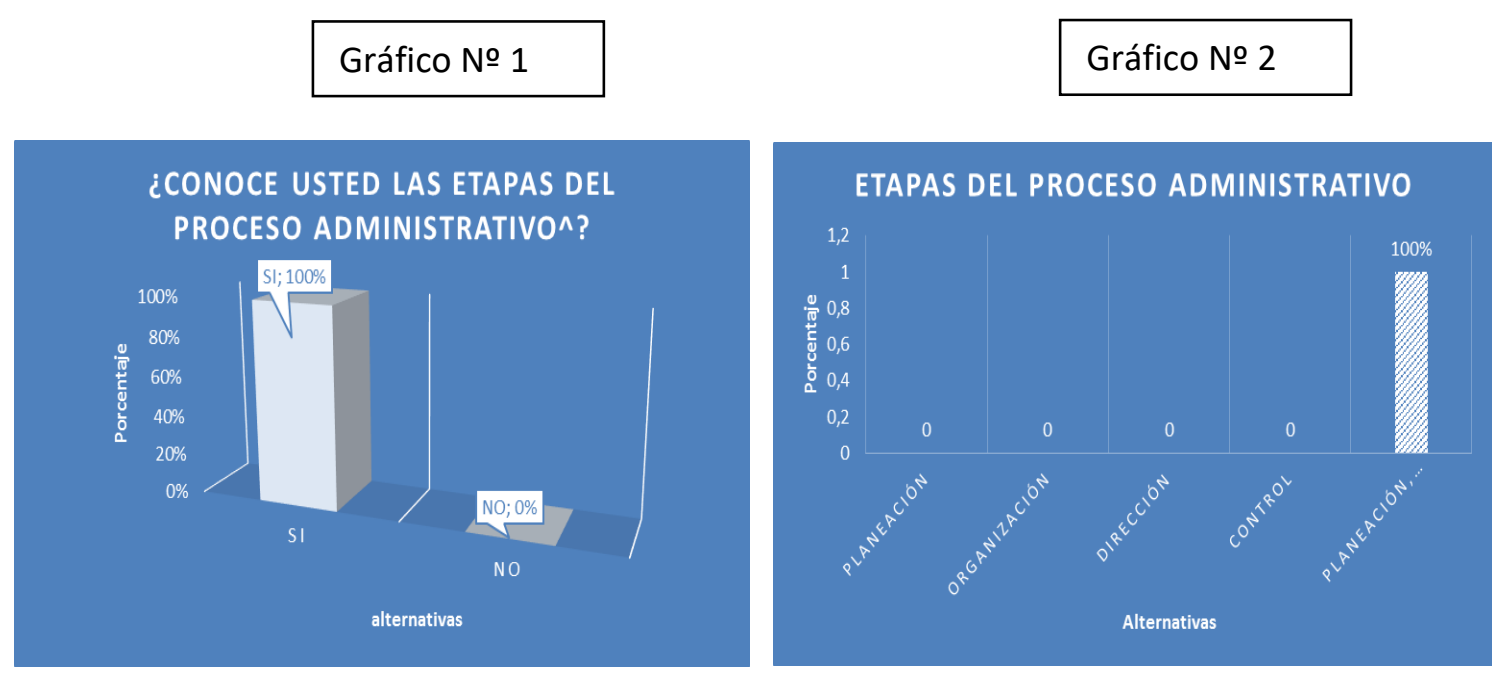

Después de tabular las respuestas obtenidas en la encuesta aplicada al personal administrativo de las unidades educativas, se manifiesta que el $100 \%$ de los encuestados responde que conocen y aplican las etapas del proceso administrativo en su totalidad, que cada una de ellas tiene su accionar y esto se basa de acuerdo a las actividades que la institución programe en el Plan Educativo de Desarrollo Institucional (PEDI), sin embargo indican que hay déficit de conocimientos en esta área y que es de suma importancia comenzar con asesoramiento, ya que con el juicio de causa se aplicarán de manera correcta las etapas para lograr resultados satisfactorios.

\section{Gráfico № 3}

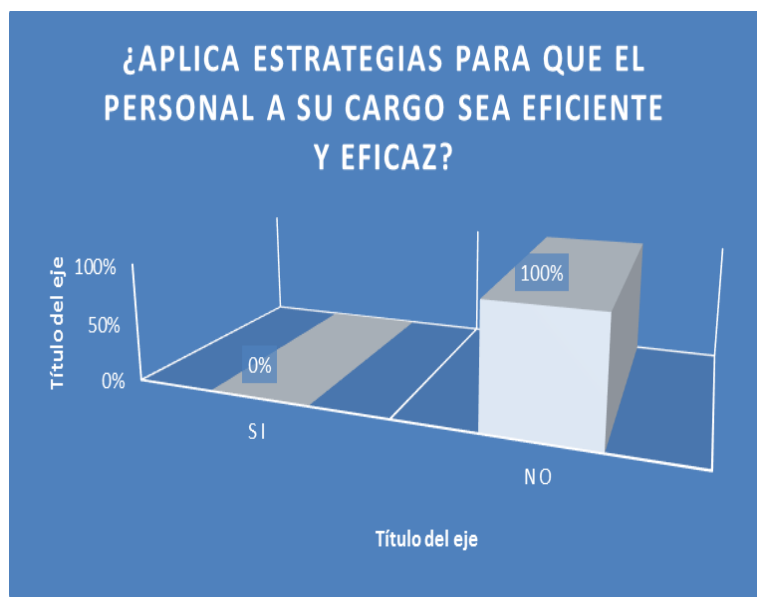

Gráfico № 4

¿QUE FACTORES APLICA PARA LOGRAR EL MÁXIMO DE SATISFACCIÓN Y PRODUCTIVIDAD EN SUS COLABORADORES?

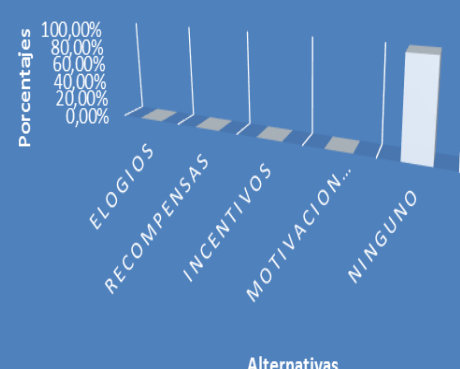


Los administrativos manifiestan que no aplican estrategias, ni hacen uso de los factores fundamentales para lograr eficiencia, eficacia, y obtener al máximo la satisfacción y productividad de sus colaboradores, porque no cuentan con el apoyo del distrito de educación, del personal que labora en la entidad, no existe un modelo rector de actividades, tampoco una planificación estratégica que visione como debe estar la unidad educativa en los próximos años.

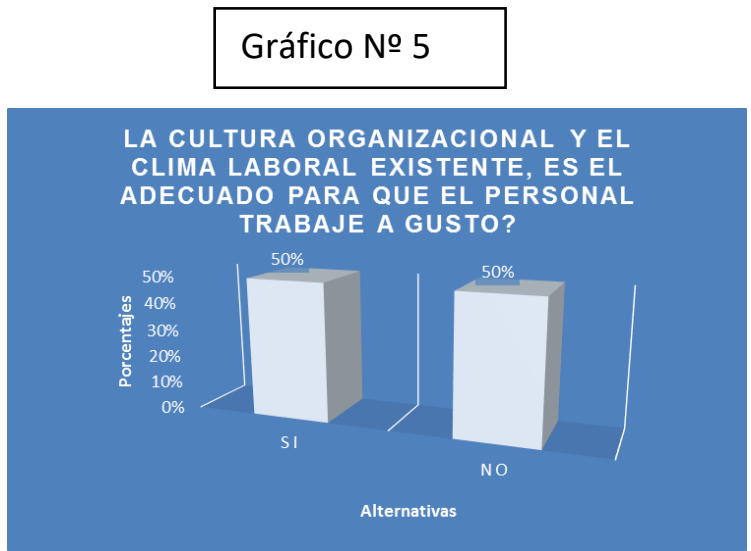

El 50\% de los encuestados responde que sí existe una cultura y un clima organizacional idóneo, mientras que el 50\% restante indica que no existe ninguno de los dos, como se puede observar existen criterios diversos, ya que cada directivo, administra según su experiencia, gestión, necesidades y de acuerdo a los activos que poseen.

Por lo que se concluye que el personal administrativo conoce las etapas del proceso administrativo, pero desconocen en un 50\% su utilización y aplicación.

\section{ENCUESTA APLICADA AL PERSONAL DOCENTE Y DE SERVICIO DE LAS UNIDADES EDUCATIVAS}

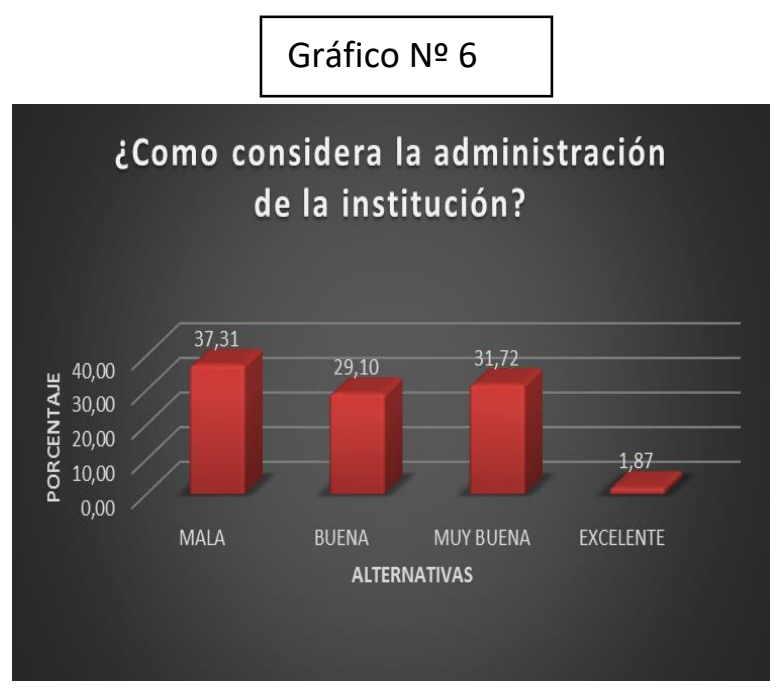


Del $100 \%$ del personal encuestado que labora en la institución el 37,31\% considera que la administración es mala, el 29,10\% que es buena, el 31,72\% que es muy buena y el1,87\% que corresponde a un pequeño número de colaboradores indica que es excelente, por ello se puede indicar que unidades educativas de la Zona 4 del Sur de Manabí, tienen como directivos apersonas que no conocen en su mayor parte sobre procesos administrativos, más bien son pedagogos y técnicos, lo que hace que no administren de forma correcta la institución y esto genera que les brinden un trato digno a los trabajadores para que se sienta satisfechos y produzcan al $100 \%$ en las actividades encomendadas.

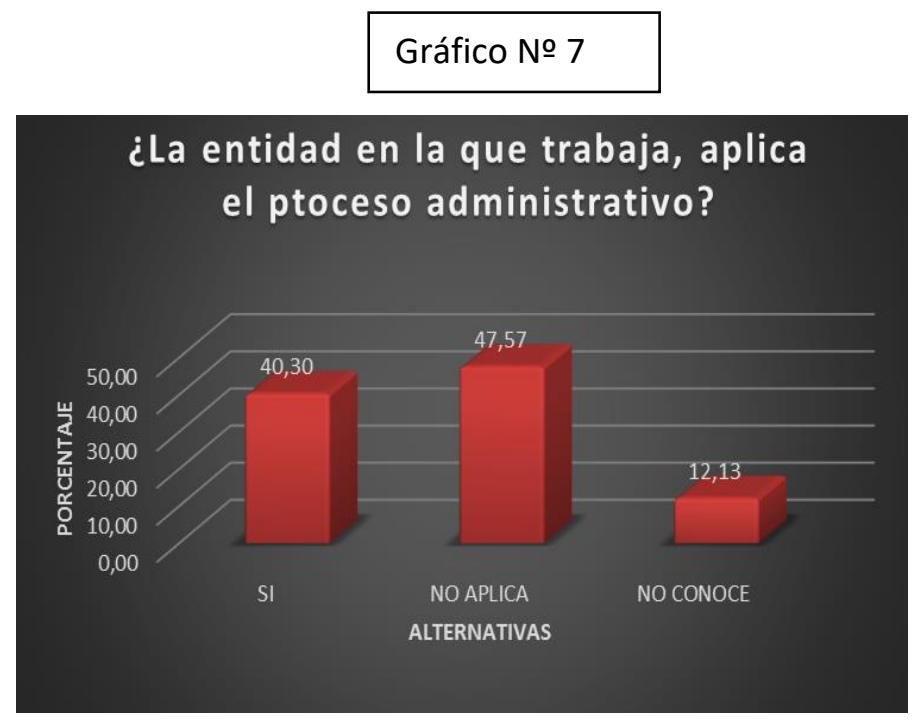

El 40,30\% de los encuestados, manifiestan que los directivos si aplican el proceso administrativo, pero es lógico puntualizar que el porcentaje de respuestas positivas esta seccionado ya que unos dicen que aplican la planeación, otros la organización, un tercer grupo la dirección y un grupo mayoritario el control, pero únicamente en la asistencia de los docentes, además un porcentaje menor dice que los directivos aplican todas las etapas del proceso administrativo, el 47,57 responde que en la entidad no se aplica el proceso administrativo y el $12,13 \%$ no conoce sobre la administración y mucho menos ha escuchado hablar del proceso administrativo.

Con los resultados obtenidos se puede apreciar que los directivos aplican en parte el proceso administrativo y por ello no aplican los subsistemas que tienen relación con el buen desempeño del talento humano, para lograr la productividad laboral absoluta. 


\section{Gráfico № 8}

¿Cual de los siguientes modelos de gestión,

aplican los directivos de la institución?

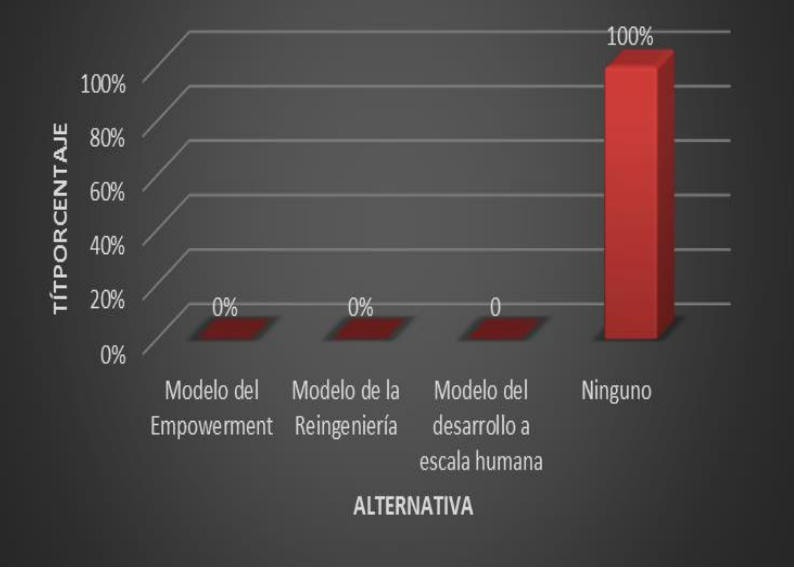

En esta interrogante se obtiene como respuesta que el100\% de los encuestados responde que los directivos no aplican ningún modelo de gestión organizacional y que nunca han escuchado hablar del tema, que únicamente conocen el modelo de gestión educativo.

Por las respuestas emitidas se puede notar que no tienen conocimientosobre la administración de la entidad, que por ello no existe adelanto en las instituciones, que no tienen autonomía y que aplican los documentos legales que les entrega el distrito de educación. Los docentes y administrativos solicitan se les capacite en temas sobre administración de empresas.

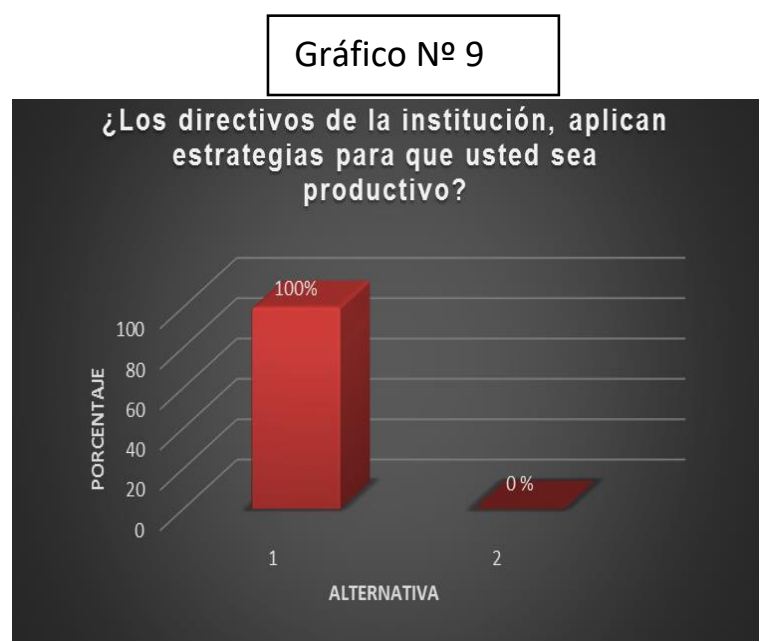

El resultado de esta pregunta es que las autoridades institucionales, no aplican estrategias para generar cambios en sus colaboradores, que siguen aplicando los métodos tradicionales, que no existe incentivo, ni motivación para provocar cambios en el personal. 
Cada persona trabaja de acuerdo a su interés por sacar adelante a la institución, siguiendo los objetivos generales de la educación media.

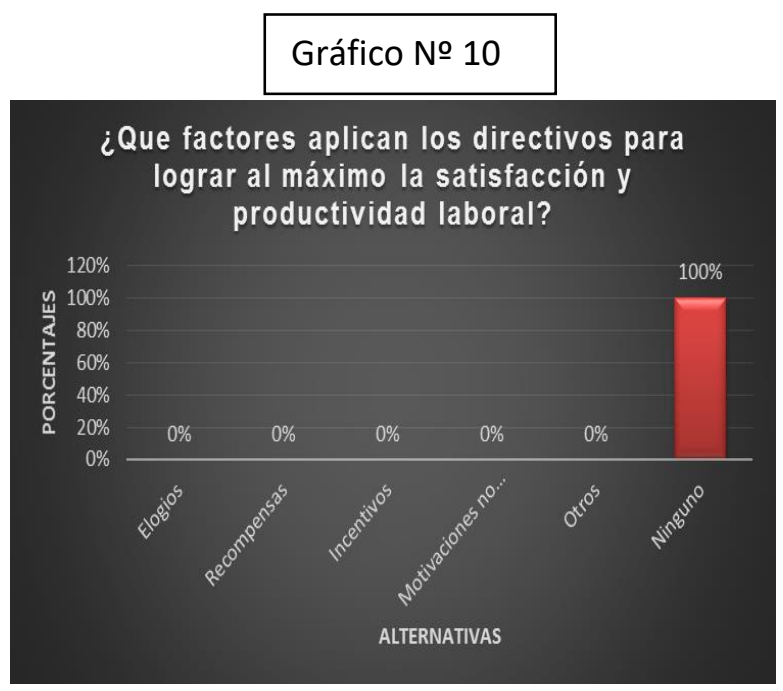

El $100 \%$ de los encuestados responde que los directivos, no aplican ningún factor para lograr la satisfacción y productividad laboral.

Lo que se aplica en la institución, es un control excesivo de los horarios de ingreso y salida, asi como en los cambios de horas clases, las reuniones u otras actividades que se realicen en la institución, se sienten perseguidos, acosados, pero no estimulados, peor motivados.

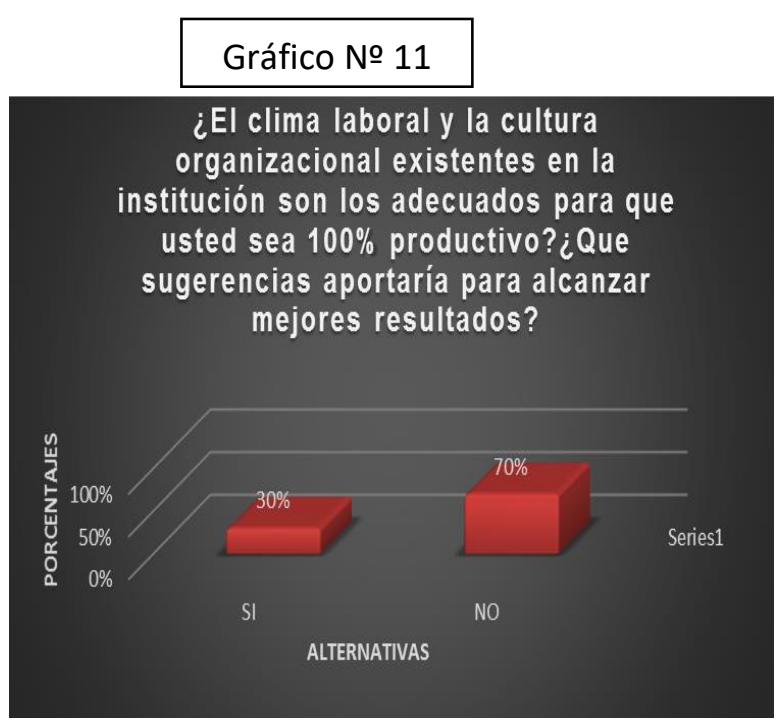

El clima laboral y la cultura organizacional, son elementos imperantes para el desempeño laboral, se los analiza en esta investigación y se obtiene que, de los encuestados, el $70 \%$ manifiesta que en la institución de trabajo no existe un ambiente laboral adecuado para trabajar, mientras que el 
30\%respondequesi existe un clima laboral acorde para el desarrollo de las actividades.

Además, como sugerencias de manera unánime solicitan lo siguiente:

Que no se conformen grupos de trabajo aislados.

Que exista un dialogo permanente

Que se detecten problemas existentes

Que se realice una distribución de trabajo adecuada

Que exista mejor comunicación

Que prime la armonía en el lugar de trabajo

Que se socialicen los modelos de gestión organizacionales

Que se realice mensualmente una rendición de cuentas

Que se generen planes de capacitación continua

Que se desarrolle y aplique una planificación estratégica

Que se genere un plan de incentivos

Que se distribuya el trabajo según la experiencia laboral y el título que obstente

Que las responsabilidades no sean direccionadas

Que en las actividades se involucre y comprometa a todo el personal

Que se les informe sobre las reformas o dediciones del Distrito y/o del Ministerio

Que se propicie la creación de un verdadero clima laboral y organizacional

Que se les de autonomía

Que se equipen las oficinas con tecnología de punta

Que se adecuan correctamente los ambientes administrativos.

Que no exista egoísmo

Que los compañeros sean solidarios

Que se mejore el estado de las aulas

Horarios flexibles

Trato igualitario

Que se aplique la cordialidad

Incremento de las TICS. 


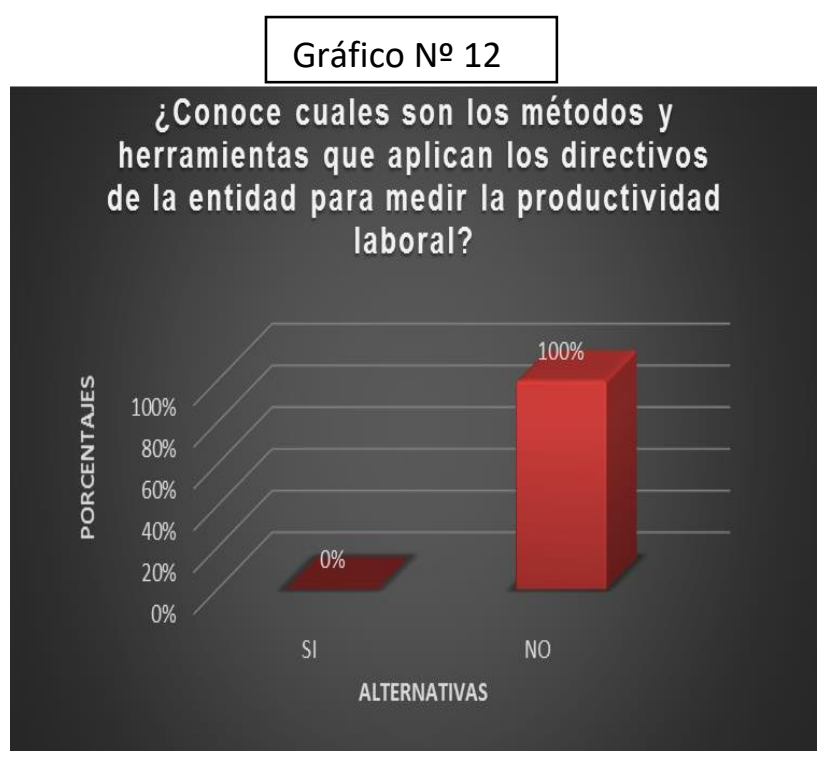

El 100\% de los encuestados indica que en la Institución que ellos laboran los directivos no aplican métodos ni herramientas para medir la productividad laboral, que aprecian su cumplimiento con la atención a los estudiantes, al llenado de leccionarios, a la entrega de portafolio, a la asistencia a clases, entre otros aspectos, por lo mencionado los encuestados dicen que los directivos no conocen a ciencia cierta la realidad de cada persona.

Con respecto a la entrevista con los rectores de las unidades educativas objeto de estudio, se obtuvo como respuesta que no tienen formación en el área administrativa, pero que conocen de la aplicación de la administración.

En lo referente a elaboración y aplicación de estrategias para crear un ambiente laborar agradable y productivo, mencionan que realizan todo de la mejor manera y que su personal está satisfecho con la administración.

\section{CONCLUSIONES:}

- Las unidades educativas de Jipijapa del sur de Manabí, están dirigidas por profesionales capaces, pero no conocen al 100\% como se conlleva la administración de una entidad, ya que aplican en parte los procesos administrativos, no conocen el modelo de gestión organizacional que presenta el Ministerio de educación, no motivan a sus colaboradores, no propician el verdadero trabajo en equipo, no existe una correcta vía de comunicación.

- El personal docente tiene mucho que entregar, pero en las condiciones que trabajan, no tienen la motivación suficiente para seguir adelante, ya que se encuentran con salones de clases que no están equipados correctamente para el dictado de las clases, compañeros con cierto grado de 
egoísmo para colaborar en actividades de la unidad educativa, horarios inflexibles, la tecnología deficiente y esto no permite la productividad total del docente. 


\section{REFERENCIAS BIBLIOGRAFÍCAS}

Acedo, J. C. (2011). Formación y Orientación Laboral. Paraninfo. . Obtenido de http://repo.uta.edu.ec/bitstream/handle/123456789/1376/296\%20Ing.pdf?sequence=1

Avalos, R. M. (2021). Gestión del conocimiento y productividad de una empresa constructora del Perú. South Florida Journal of Development, Miami, 5183 - 5194.

Blanco, Samano, Barrelleza, S. B. (2018). Elementos básicos de administración. Mexico: Servicios editoriales Veinte Rios.

Castro, M. C. (2021). Propuesta de un modelo pedagógico e-learnig en la universidad de san martín de porres. South Florida Journal of Development, Miami, 5787 - 5804.

Carraco y Viñan (2017). Elementos de ingenieria administrativa. Riobamba - Ecuador: Editorial Politécnica ESPOCH.

Chiavenato, I. (2008). Introducción a la teoria general de administración.

Chiavenato, R. -I. (1954 - 2010). The practice of management - Gestión del talento humano. New Yoek: Mc Graw Hill.

Contreras Soto, R. (2011). Reflexiones en torno a la construcción científica en el campo de los estudios de las organizaciones, gestión y administración en México. Observatorio de la economia latinoamericana, 20-56., 20-56.

Direccicón general de productividad laboral, G. F. (2012). Sistema de Gestión para la productividad Laboral. México, México, México

Ecuador, M. d. (10 de 10 de 2017). Guia de evaluación práctica para aspirantes a autoridades educativas. Quito, Pichincha, Ecuador: Transformar educación

Ecuador, M. d. (2017). Manual para la Implementación y Evaluación de los Estándares de Calidad Educativa. Quito: Medios públicos.

Ecuador, A. N. (2008). Documento. Obtenido de Constitución de la República de Ecuador.

Gabini, S. (2018). Potenciales predictores del rendimien tolaboral. Buenas Aires: Teseo.

Koonts, H. W. (2012). Libro: Administración una perspectiva global y empresarial. México: McGrawHill. Interamericana Editores S.A. 
Lopez. (2007). Teologia e histotia. Obtenido de https://csalazar.org/.../historia-de-la-administracion-enlas-antiguas-civilizaciones-3/

Maslow, A. (1957- 2007). documento. Obtenido de Comportamiento Organizacional.

Meter, E. W. (2012). Ladrones de tiempo. Obtenido de www.workmeter,com

Munch, L. (2014). Gestión organizacional, enfoques y proceso administrativo - segunda edición. Mexico: PEARSON.

Mundial, B. (2017). Informe Anual. Washington DC - USA: Grupo Banco Mundial . Organización de las Naciones Unidas para la Educación, 1. C.

Ramirez, C. (2015). Fundamentos de Administración. Bogota Colombia: Ecoe Ediciones.

Taylor, F. (1969). Principios de la Administración Científica (11ºdición). México: Herrero Hnos. S. A. UNESCO. (2017). Modelo de Gestión. Santiago, Santiago, Chile.

Velázquez, M. (15 de 08 de 2016). 5 factores que afectan la productividad en el trabajo. Obtenido de Entrepreneur: https://www.entrepreneur.com/article/280867 